\title{
Auto-doffer* as a Means of Automation
}

\author{
By Yorikazu Shimotsuma and Kazuhiko Sakaguchi, Members, TMSJ
}

\author{
Faculty of Engineering, Kansai University, Osaka
}

\section{Introduction}

It is common knowledge that a feed back system, a digital system, a sequence system, etc. are used to control an automatic producing machine. Many controlling apparati have been used in the textile industry since long before the systematization of control engineering. For example, the piano-motion of an opening and beating machine is a feed back control system. The Jacquard apparatus of a loom is a sequence control system. These apparati have been widely used since long before the concept of feed back control or sequence control was established.

An auto doffer is a very interesting apparatus from the view point of control engineering. Highly complex movements are required of an auto doffer. Many types of control systems, specially sequence control systems, are combined in an auto doffer. An auto doffer is required to work to full capacity and without a respite. Nor must it get out of order. If it does, it stops all operations in a spinning mill. An auto doffer must, therefore, be durable, reliable and safe to use.

Three auto doffers, each differing entirely in build and mechanism, have been perfected in Japan. They are already in service. Researches for improvements are in progress.

The history of the development of auto doffers in Japan and abroad shows that individual ideas conceived and developed have given incentives and suggestion - and led to cooperation, national and international, in research and development. This is a notable feature of the development of auto doffers.

Japanese auto doffers have won international recognition as being of the highest standard. As a part of continuous, automatic spinning equipment or as a separate item, Japanese auto doffers have played a big part in bringing the level of the Japanese textile and textile machinery industries anew to the notice of the world.

\section{Progress toward Automation}

Mechanization and, then, automation of labor is an unavoidable task in the progress of all industries, but the task is fraught with multiple problems. Mechanization and automation certainly reduce the need for manual labor, but they also reduce the versatility of machines.

Therefore, automation is inadvisable unless the machine involved is a mass-production machine. High-degree automation reduces a machine's adaptability to changes in operating conditions. Highdegree automation, again, needs high-degree control of the machine by skilled experts and can defeat the aim of automation - reduction of the need for manual labor. We may say generally that the further a machine is automated, the more "specialized" and complicated it becomes.

All this holds true of auto doffers. It should be borne in mind in all attempts at further improvements in auto doffers.

\section{Classified Existing Doffers and Their Features}

Novel ideas, new technologies, trial designings and research work have given birth to a wide variety of auto doffers. We will classify them by models and their features. A common method of classification or common names for the various models are unobtainable, because the existing auto doffers are still undergoing efforts at further improvements. In this article we use the following classifications:

\section{(1) Classification by builds}

I. Crane type

A crane-type auto doffer is a big item of equipment consisting of an overhead travelling crane with a cop-holding apparatus and a bobbin-holding apparatus attached to it. The crane doubles as a conveyor of slivers and bobbins.

A crane-type auto doffer enables the doffing of all spindles at one time. This means a relatively short time for spindle doffing and, therefore, high efficiency. This doffer, however, entails heavy installation costs. For one thing, rails for the heavy, big crane has to be laid at a high place. 
This disadvantage, however, is offset considerably by the fact that one doffer takes charge of numerous spindles and does carrying and cleaning also.

Doffing and donning work is extremely simple, because cops and bobbins are doffed and donned from above. Simple in mechanism, the crane type works with a high degree of accuracy.

\section{Wagon type}

This type is featured by a doffing wagon which moves in front of the frame and does doffing in sequence. Therefore, the wagon must be loaded with empty bobbins and must have a receptacle for cops. The wagon type is based on shift-system manual labor and is an effort to mechanize it.

(1) Intermittent-moving wagon type

The wagon stops at a fixed place in front of the frame and does doffing while standing there. Doffing completed, the wagon moves to another fixed place to do doffing. In other words, the wagon repeats a stop-go-and-stop cycle.

Normally, only the front half of the wagon (in the direction in which the wagon is going) doffs cops. The rear half only dons bobbins. Therefore, the wagon unavoidably needs a cop-doffing device and a cop-donning device.

Doffers of this type mostly use the cam mechanism and, therefore, presents mechanical problems unique to the cam mechanism, as well as the problem of timing.

(2) Continuous-moving wagon type

The wagon doffs the spindles one by one as it moves continually in front of the frame. This type may be thought of as a combination of a straightmoving cam mechanism and a translation-cam mechanism.

The intermittent-moving type fluctuates sharply in wagon speed. Therefore, the inertia of acceleration-and is, easier to design.

\section{Frame-attached type}

This type is featured by the fact that a doffing apparatus is attached to each and every frame of a spinning machine. The cop-doffing apparatus and the bobbin-donning apparatus for each spindle are run by hand, oil pressure, air pressure or electric motor.

The main moving axis passes through all frames while it is near the ring rail. There is an arm for each spindle. Beyond the arm is a doffing apparatus. There are various other devices provided. It seems this type is intended for many types of work besides doffing.

\section{(2) Classification by functions}

Many auto doffers, for technical or economic reasons, does other work besides doffing. This is an interesting fact because it seems to suggest the future trend. Meanwhile, this article assumes that the work of a doffer is limited to:

$\begin{array}{ll}\text { [Doff]: } & \text { Doffing } \\ \text { [Don]: } & \text { Donning } \\ \text { [Trans.]: } & \text { Transportation } \\ \text { [Clean]: } & \text { Cleaning } \\ \text { [Wind]: } & \text { Winding }\end{array}$

1) $[$ Doff $]+[$ Don $]$

This is the minimum work required of a doffer. It is the commonest type. Many wagon-type doffers come under this classification. So do some doffers of the frame-attached type.

2) $[$ Doff $]+[$ Don $]+[$ Trans $]$

The crane type falls under this classification. Some doffers of the frame-attached type do doffing by collecting cops on a conveyor.

3) $[$ Doff $]+[$ Don $]+[$ Trans $]+$ [Clean $]$

The crane type with a cleaning apparatus attached comes under this classification.

4) $[$ Doff $]+[$ Don $]+[$ Wind $]$

This is a combination of a spinning machine and a winder. It is a newcomer.

\section{Classification by Number of Spindles (the commonest method of classification)}

\section{(1) One-spindle system}

The wagon type needs only a small wagon, but it is extremely limited in doffing time and, therefore, poses difficulties in designing and building.

The frame-attached type on the rail-travel system has more doffing time and is, therefore, easier to design.

\section{(2) Multi-spindle system}

This system runs several spindles per cycle to relax the rigid time limit imposed by the one-spindle system. Many wagon-type doffers come under this classification. If there is a large number of spindles for simultaneous doffing, the time needed per cycle is long almost in proportion to the number of spindles. This is good, but then a large wagon is needed, more power is needed and costs increase. Therefore, the optimum number of spindles depends on the doffing mechanism used and the operating conditions. 


\section{(3) Continuous system}

The continuous moving type comes under this category. Both the single-spindle system and the multi-spindle system impose a limit on doffing time. With the continuous system it is difficult to fix the cam profile curve. The effort to operate the follower smoothly reduces the pressure angle of the cam. The smaller the pressure angle of the cam, the longer the translation cam and, therefore, the larger an auto doffer in size. To cope with these two opposing conditions, we have to have an optimum designing of an auto doffer of the continuous system type.

\section{(4) All-spindles system}

Falling under this category are the crane type and the frame-attached type operated on the allspindles system.

\section{Auto Doffers as Automated Machines}

Generally, automation of a productive machine requires separate programs for the individual movements of the machine. By "programs" we mean movement control drums.

An auto doffer, however, needs only a series of set movements. It does not require a wide variety of movements as does a machine tool. The movements of an auto doffer as an automated machine are simplified. This is presumably one of the reasons why auto doffers have been brought to the present stage of development speedily.

Generally, progress in automation is gradual. It goes through stages. Looking at the existing auto doffers, we find that in their initial stage, they merely replaced the hand with the machine. Now they have been automated in a large degree sectionally. The movements of various parts of an individual auto doffer disclose stages in the progress of auto- mation. This is a matter of course in the progress of automation in all fields, but it is an interesting aspect of auto doffers as automated machines.

An auto doffer must never go out of order. An accident to an auto doffer stops thousands or tens of thousands of spindles and the other spinning equipment. An auto doffer must always work accurately and reliably - and must be equipped with accident prevention devices, an alarm signal device and an automatic stopping device. This is where an auto-doffer differs from other automated machines.

\section{Conclusions}

The author may incur the charge of discussing only three types of auto doffers: Crane, wagon and frame-attached. Further improvements in them are being planned for operational and economic reasons. Efforts are in progress to re-design the crane type so that it will do transportation and cleaning also and take care of more frames - for reasons of economy.

Efforts are being made to re-design the wagon type for higher efficiency and a higher degree of reliability while retaining its outstanding advantage of requiring no major changes in an existing mill or its spinning equipment. An idea is taking shape to connect the frame-attached type with a winder by a conveyor.

These different types of auto doffers, each, have distinct characteristics. Selection of any type should depend on its technical features, the mill operation economy and the stage of automation in the mill.

* The term "auto doffer" used in this article means, in the main, an automatic doffing-donning machine attached to a fine spinning machine. 